\title{
Tumoricidal activities of pterostilbene depend upon destabilizing the MTA1-NuRD complex and enhancing $P 53$ acetylation in hepatocellular carcinoma
}

\author{
YU-YUAN QIAN ${ }^{1}$, ZHI-SU LIU ${ }^{2}$, DING-YU PAN ${ }^{2}$ and KUN LI ${ }^{1}$ \\ Departments of ${ }^{1}$ Hepatobiliary Surgery and ${ }^{2}$ Pancreatic Surgery, \\ Zhongnan Hospital of Wuhan University, Wuhan, Hubei 430071, P.R. China
}

Received March 15, 2017; Accepted July 20, 2017

DOI: $10.3892 /$ etm.2017.4923

\begin{abstract}
The present study aimed to assess the tumoricidal effect of metastasis-associated protein 1 (MTA1) induced by pterostilbene (PTER) in hepatocellular carcinoma (HCC). The SMMC-7721 hepatoma cell line was treated with PTER. Following treatment, the mRNA transcript abundance of MTA1 was measured using quantitative polymerase chain reaction. Additionally, cell viability was determined using an MTT assay, and protein expression was measured through western blotting. Cell invasion, motility and apoptosis, as well as the cell cycle, were also investigated. Following PTER treatment, MTA1, histone deacetylase (HDAC) 1 and HDAC2 were downregulated, whereas the ratio of acetyl-p53 to total p53 was increased in HCC cells. Cell viability decreased as the PTER dose increased. MTA1 may be associated with proliferation, motility, invasion and metastasis in HCC cells PTER appeared to repress cell proliferation, trigger apoptosis, induce cell cycle arrest, and inhibit motility and invasion via MTA1 in human liver cancer cells. The results of the present study demonstrated that PTER can downregulate the MTA1-nucleosome remodeling and deacetylase complex, and enhance p53 acetylation to inhibit the growth of tumor cells in HCC.
\end{abstract}

\section{Introduction}

Hepatocellular carcinoma (HCC) is one of the most common types of tumors and is the most frequently occurring type of liver cancer, leading to over 700,000 deaths each year worldwide. Conventional chemotherapy has minimal therapeutic effects on liver cancer, and its diagnosis is often too late

Correspondence to: Dr Kun Li, Department of Hepatobiliary Surgery, Zhongnan Hospital of Wuhan University, 169 Donghu Road, Wuhan, Hubei 430071, P.R. China

E-mail: qianyy888@sina.com

Key words: MTA1, pterostilbene, hepatocellular carcinoma, HDAC, P53 achieve the optimal operation time, resulting in the rapid death of affected individuals $(1,2)$.

The expression of metastasis-associated protein 1 (MTA1) is common in many types of cancers. Previous studies have shown that the MTA1 protein, along with the histone deacetylase 1 and 2 (HDAC1 and HDAC2) complex, are members of the nucleosome remodeling and deacetylation (NuRD) protein complex. MTA1 belongs to the MTA protein family that contributes to the NuRD protein complex and contributes to the regulation of specific genes, mediates histone deacetylation, and remodels chromatin $(3,4)$. We recently discovered that pterostilbene (trans-3,5-dimethoxy-49-hydroxystilbene, PTER) downregulates MTA1, resulting in the inhibition of tumor growth. PTER is a stilbenoid that is found in blueberries and grapes and plays an important role in inhibiting cancer growth, reducing hyperlipidemia, and treating cognitive decline $(5,6)$.

However, the clinical significance of the MTA1-NuRD complex and p53 acetylation and their role in the tumoricidal effects induced by PTER for HCC merit further research. In this current study, we cultured SMMC7721 cells and treated them with different doses of PTER. We investigated protein expression levels in these cells by western blotting and cell viability by MTT assay. Cell functions were investigated by invasion, motility, apoptosis, and cell cycle assays. Our study reveals that MTA1 is a major PTER target that may have therapeutic effects and potential applications in HCC.

\section{Materials and methods}

Cell culture. We obtained the SMMC-7721 hepatoma cell line from the China Center for Type Culture Collection (Department of Medicine, Wuhan University, Wuhan, China). Cells were maintained in humidified air with $5 \% \mathrm{CO}_{2}$ at $37^{\circ} \mathrm{C}$ and cultured in RPMI-1640 medium (Gibco, Grand Island, NY, USA) with $10 \%$ heat-inactivated fetal bovine serum, $100 \mathrm{U} / \mathrm{ml}$ penicillin, and $100 \mathrm{mg} / \mathrm{ml}$ streptomycin. Cell viability was generally greater than $98 \%$ prior to treatment.

Quantitative polymerase chain reaction ( $q P C R)$ analysis. We extracted total RNA from SMMC7721 cells using TRIzol (Invitrogen, Paisley, UK) according to the manufacturer's 
instructions. A spectrophotometer was used to quantify the concentration of total RNA based on absorbance at $A_{260}$ and $A_{280}$. Total RNA was reverse transcribed using the SuperScript III platinum two-step RT-qPCR kit with SYBR-Green (Invitrogen Life Technologies, Carlsbad, CA, USA). Next, qPCR was used to analyze mRNA transcript abundance with the MiniOpticon Real-Time PCR system (Bio-Rad, Berkeley, CA, USA). PCR primers were as follows: MTA1 forward, 5'-CGCTGACCA GCATCATTGAGT-3'; and reverse, 5'-TGGTTCGGATTT GGCTTGTTAT-3', $\beta$-actin forward, 5'-CGTGGGCCGCCC TAGGCACCA-3', and reverse 5'-TTGGCTTSGGGTTCSG GGGGG-3'. Transcript quantification and analysis were performed using Bio-Rad CFX Manager v3 software, and calculations were carried out using the the $2^{-\Delta \Delta C q}$ method. Data are presented as the means \pm standard deviation of three independent experiments.

Western blot analysis. Western blotting was carried out as described previously (7). Briefly, SMMC-7721 hepatoma cells were treated with various concentrations $(5,25,50$, or $100 \mu \mathrm{M})$ of PTER for $24 \mathrm{~h}$. PTER (Sigma, St. Louis, MO, USA) was dissolved in dimethyl sulphoxide (DMSO); DMSO alone was used to treat cells in the control group. After washing with PBS, cells were then lysed using RIPA buffer, which was mixed with the Halt Phosphatase and Protease Inhibitor Cocktail (Thermo Fisher Scientific, Inc., Waltham, MA, USA). After the protein concentration was quantified, $70 \mu \mathrm{g}$ of protein was resolved by SDS-polyacrylamide gel electrophoresis (PAGE) and transferred to a PVDF membrane using an Electrophoresis Transfer System (Bio-Rad). Subsequently, membranes placed on a swing table were blocked with TBS-Tween and 5\% dry milk and were then probed with anti-MTA1, p53 or Ac-p53 (Abcam, Cambridge, UK) primary antibodies overnight at $4^{\circ} \mathrm{C}$. Respective secondary antibodies (Abcam) were used at 1:1,000-1:2,000 (v/v) dilutions in TBS-Tween and 5\% fat-free powdered milk for $1 \mathrm{~h}$ at room temperature; $\beta$-actin antibodies were used as a loading control. Finally, blots were visualized by SuperSignal West Pico enhanced chemiluminescence substrate (Thermo Fisher Scientific, Inc., Waltham, MA, USA), and the density of the blots was quantified using Image J Software (Bio-Rad).

MTT assay. To test cell viability, we used the MTT assay with the cell proliferation reagent MTT [3-(4,5-dimethylthiazol-2-yl)-2,5-diphenyltetrazolium bromide]. After PTER treatment, $\sim 20 \mu \mathrm{l}$ of sterile filtered MTT (Sigma) stock solution in phosphate-buffered saline, $\mathrm{pH} 7.4$, was added to each well. After cells were incubated for $\sim 4 \mathrm{~h}$, media was aspirated to remove unreacted dye, and $\sim 150 \mu 1$ dimethylsulfoxide (Sigma) was added; cells were then shaken for $10 \mathrm{~min}$. Absorbance at $490 \mathrm{~nm}$ for each sample was measured in a 450 microplate reader (Bio-Rad). The spectrophotometer was calibrated to zero absorbance with culture medium without cells. We calculated the relative cell viability (\%), normalized to the control cells, as follows: [(test quantitative value)/(control quantitative value)] x $100 \%$. A total of three independent experiments were performed with three wells per experimental condition.

Flow cytometric analysis of the cell cycle. SMMC7721 cells were fixed with $70 \%$ ethanol overnight at $4^{\circ} \mathrm{C}$ and were collected the next day and washed twice with PBS. Then, cells were treated with $0.5 \mathrm{mg} / \mathrm{ml} \mathrm{RNase} \mathrm{(Sigma)} \mathrm{for} 30 \mathrm{~min}$ and stained with propidium iodide (PI) using a Cell Cycle Detection kit (Sigma) to analyze the cell cycle at room temperature in the dark for $30 \mathrm{~min}$ according to the manufacturer's instructions. We counted 14,000 events for each PI-stained sample on a FACS Calibur (BD Biosciences, Franklin Lakes, NJ, USA) and analyzed the percentage of cells in the G1, S, and G2/M phases using a FACScan (BD Biosciences, San Jose, CA, USA) flow cytometer. ModFit LT version 3.2 software (Verity Software House; Topsham, Devon, UK) was used to analyze the cell cycle data.

Flow cytometric analysis of apoptosis. SMMC7721 cells were stained with Annexin V-FITC and PI using an Annexin V-FITC Apoptosis Detection kit (Sigma) to analyze the percentage of apoptotic cells according to the manufacturer's instructions. Stained cells were analyzed on a flow cytometer and calculated using FlowJo V10 software (BD Bioscience).

Cell invasion assay. The transwell invasion assay was used to test invasion capability of SMMC7721 cells. Cells were placed in the upper transwell chamber (8 $\mu \mathrm{m}$ pore size; Corning Incorporated, Corning, NY, USA) coated with Matrigel (BD Biosciences, Franklin Lakes, NJ, USA), and $600 \mu \mathrm{l}$ of the test medium was added to the lower chamber. After cells had been incubated at $37^{\circ} \mathrm{C}$ and $5 \% \mathrm{CO}_{2}$ for $24 \mathrm{~h}$, non- invasive cells that adhered to the upper membrane were wiped off using a cotton swab. Migrated cells were stained with crystal violet dye, fixed with methanol, and viewed using a microscope.

Wound healing assays. A wound healing assay was used to detect the motility capacity of SMMC7721 cells. Prior to wounding, $5 \times 10^{5}$ cells were seeded in six-well plates and incubated for $24 \mathrm{~h}$ with serum-free RPMI-1640. A $10 \mu \mathrm{l}$ pipette tip was used to scratch the monolayer and create a 'wound' across the diameter of the each well. Then, detached cells were removed by a gentle wash with PBS. Cells were incubated at $37^{\circ} \mathrm{C}$, allowing cells to migrate across the open wound area. A light microscope (Olympus Corp., Tokyo, Japan) was used to image wound areas at appropriate time points $(3,6,9,12$ and $24 \mathrm{~h})$. Experiments were conducted in triplicate.

Statistical analysis. Each experiment was repeated at least three times. All experimental results and measurements are expressed as the means \pm standard deviation (SD). The bivariate method and Student's t-test were used to analyze differences between groups. All histogram data were evaluated using GraphPad Prism, version 6.0 (GraphPad Software, San Diego, CA, USA). $\mathrm{P}<0.05$ was considered to indicate a statistically significant difference.

\section{Results}

PTER downregulated MTAl mRNA in a dosedependent manner in vitro. SMMC7721 cells were cultured and treated with various concentrations of PTER $(5,25,50$, or $100 \mu \mathrm{M}$ ) for $24 \mathrm{~h}$. As shown in Fig. 1A, PTER significantly downregulated MTA1 mRNA transcripts in a dose-dependent 

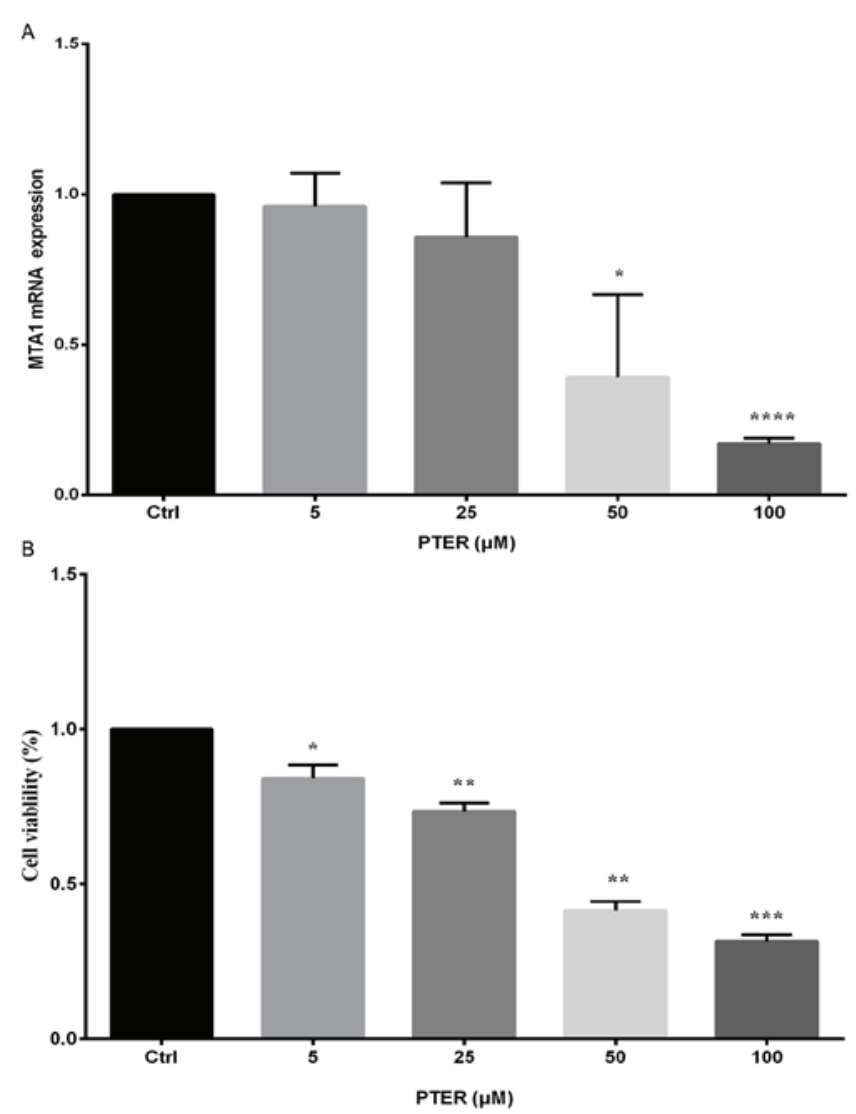

Figure 1. SMMC7721 mRNA expression and cell viability after $24 \mathrm{~h}$ treatment with different doses of pterostilbene (PTER). (A) Cells were treated with DMSO [control (Ctrl)] or PTER $(5,25,50$, or $100 \mu \mathrm{M})$ for $24 \mathrm{~h}$. Cells were harvested, and the abundance of metastasis-associated protein 1 (MTA1) mRNA transcripts was measured. Results were analyzed by real-time PCR and calculated using the equation $2^{-\Delta \Delta c q}$. The $\Delta \mathrm{Cq}$ of MTA1 mRNA transcripts was calculated by subtracting the $\mathrm{Ct}$ of $\beta$-actin from that of MTA1; then, the $\Delta \Delta \mathrm{Cq}$ equal to the $\Delta \mathrm{Cq}$ from the PTER-treated groups was subtracted from that of the $\Delta \mathrm{Cq}$ Ctrl group. (B) Cells were treated with DMSO (Ctrl) or PTER $(5,25,50$, or $100 \mu \mathrm{M})$ for $24 \mathrm{~h}$ to detect cell viability. The Ctrl was normalized to 1 , and cell viability values were calculated relative to those of Ctrl. Values represent the means \pm standard deviation from three independent experiments. Comparisons between groups were performed using unpaired two-tailed Student's t-test; ${ }^{*} \mathrm{P}<0.05,{ }^{* *} \mathrm{P}<0.01,{ }^{* * * *} \mathrm{P}<0.001,{ }^{* * * * *} \mathrm{P}<0.0001$.

manner. These findings indicated that PTER inhibited mRNA transcript expression in vitro.

PTER suppressed HCC cell proliferation. Viable cells express the mitochondrial dehydrogenase enzyme, which can reduce the yellow dye MTT, leading to the formation of dark blue formazan crystals that are largely impermeable to cell membranes and accumulate in viable cells. Results were analyzed using a multiwell scanning spectrophotometer. There were significant differences in the death rate between the PTER $(5,25,50$ and $100 \mu \mathrm{M})$ and control groups $(\mathrm{P}<0.05)$. Data shown in Fig. 1B indicate that cell viability was reduced with an increasing dose of PTER. The MTT assay results showed that $50 \mu \mathrm{M}$ PTER could induce a significant reduction in cell viability.

Inhibition of the MTA1/HDAC complex by PTER. Cells were treated with different doses of PTER for $24 \mathrm{~h}$, and protein was isolated for western blot analysis. After treatment with
PTER, the expression of MTA1, HDAC1 and HDAC2 were downregulated compared with DMSO-treated cells (Fig. 2A). PTER potently inhibited MTA1 mRNA transcript expression and showed a concentration-dependent reduction in protein levels (Fig. 2B). The reduction in MTA1 levels was shorter and less consistent than that of the MTA1/HDAC complexes. Thus, HDAC1 and HDAC2 expression could be significantly reduced $(\mathrm{P}<0.01)$ by inhibition of MTA1 (Fig. 2C and D). These findings demonstrated that PTER markedly increased MTA1-mediated p53 acetylation, implying that it played an important role in posttranslational protein modifications. PTER treatment at $50 \mu \mathrm{M}$ for $24 \mathrm{~h}$ elevated both total and Ac-p53 levels. After PTER treatment, p53 became acetylated, which induced the accumulation of stable protein. Remarkably, according to a quantitative analysis of the ratio of Ac-p53-to-total p53, the PTER treatment group exhibited a higher ratio than the controls in SMMC7721 cells (Fig. 3). In summary, PTER clearly induced the downregulation of MTA1, which increased p53 acetylation, leading to activated p53-mediated pathways in SMMC7721 cells.

PTER inhibited cell motility and invasion in SMMC7721 cells. The motility and invasion of cancer cells have been linked to cancer metastasis in vitro. In our present study, the number of cells that moved or invaded into the open surface was reduced after PTER treatment. The amount of cell motility was decreased after treatment with PTER for 3, 6, 9, 12 and $24 \mathrm{~h}$ in SMMC7721 cells (Fig. 4A and B). In the Matrigel invasion assay, we observed that PTER could markedly arrest cell invasion in SMMC7721 cells compared with the control group (Fig. 4C and D).

PTER induced cell cycle arrest in SMMC7721 cells. To further explore the anti-proliferative effects of PTER, we assessed the cell cycle profile and apoptosis of SMMC7721 cells (Fig. 5). Cells in $\mathrm{S}$ phase were significantly increased after treatment with $50 \mu \mathrm{M}$ PTER for 3 and 5 days compared with the control group. In accord with the increased number of cells in $\mathrm{S}$ phase, fewer SMMC7721 cells were in G2/M phase (Fig. 5C). We found that PTER treatment clearly increased the amount of cells in S phase (control, 3 days group: $30.55 \pm 0.75 \%$, PTER-treated, 3 days group: $38.25 \pm 1.56 \%, \mathrm{P}<0.0001$; control, 5 days group: $41.46 \pm 1.83 \%$, PTER-treated, 5 days group: $34.36 \pm 1.93 \%$ ) and decreased the amount of cells in G2/M phase (control, 3 days group: $27.12 \pm 1.77 \%$, PTER-treated, 3 days group $20.40 \pm 0.93 \%, \mathrm{P}<0.0001$; control, 5 days group: $24.04 \pm 1.59 \%$, PTER-treated, 5 days group: $17.20 \pm 1.50 \%$, $\mathrm{P}<0.0001)$ in cultured SMMC7721 cells. These days suggested that the cell cycle progression of PTER-treated cells stalled at $\mathrm{S}$ phase by inhibiting the S-G2/M phase transition compared with the control group (Fig. 5D and E).

PTER induced apoptosis of SMMC7721 cells. The induction of apoptosis was assessed after treatment with $50 \mu \mathrm{M}$ PTER for 3 and 5 days in SMMC7721 cells (Fig. 5A). Representative nuclei of apoptotic cells exhibited condensation and fragmentation. Based on Annexin V-FITC and PI staining, the frequencies of apoptotic cells were analyzed. As shown in Fig. 5B, the total frequency of apoptotic cells increased to $9.32 \pm 0.09$ and $9.24 \pm 0.19 \%$ after $50 \mu \mathrm{M}$ PTER treatment 
A
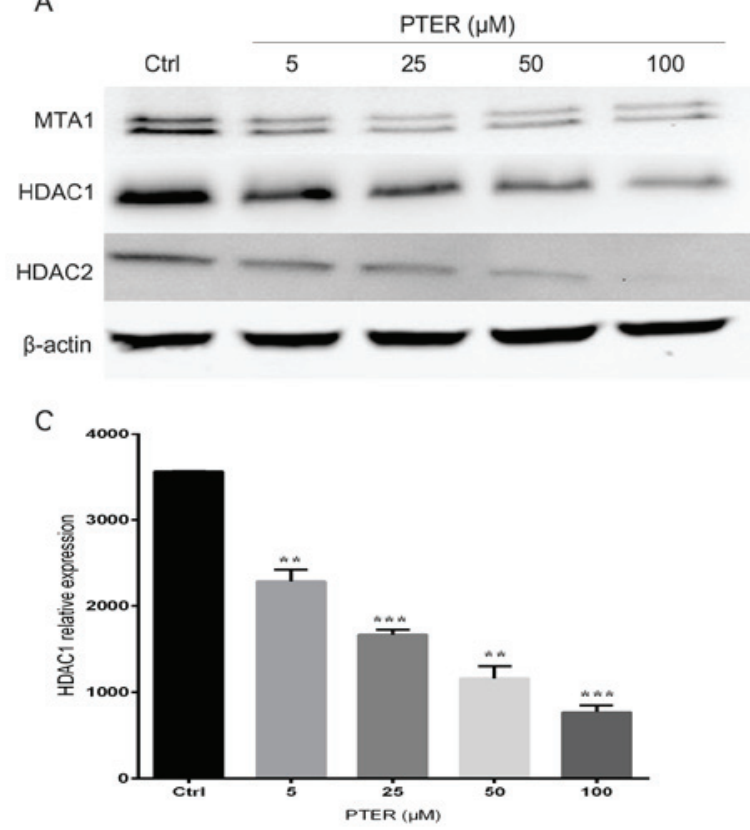

B

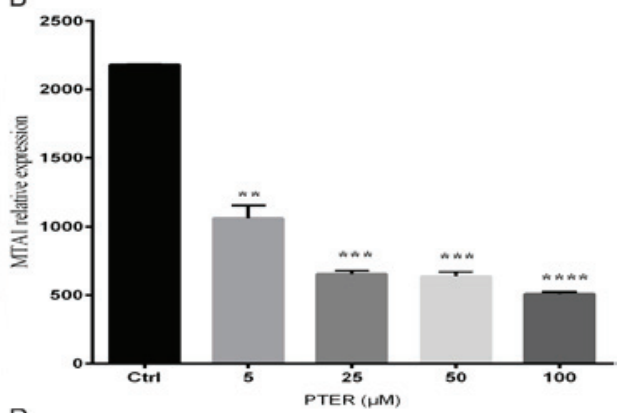

D

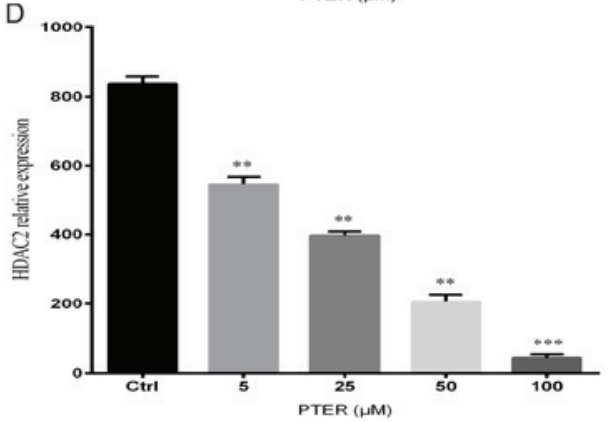

Figure 2. Inhibition of the metastasis-associated protein 1 (MTA1)-histone deacetylase (HDAC) complex by pterostilbene, which induced the downregulation of MTA1, HDAC1 and HDAC2 expression in pterostilbene (PTER)-treated cells. (A) SMMC7721 cells were treated with various concentrations (5, 25, 50 or $100 \mu \mathrm{M}$ ) of PTER for $24 \mathrm{~h}$; the control (Ctrl) group was treated with DMSO. Protein expression levels were analyzed by western blotting for MTA1, HDAC1, HDAC2 and $\beta$-actin. (B-D) Western blotting results were quantified using Image $J$ analysis software. The means \pm standard error of three independent expression are shown; ${ }^{* *} \mathrm{P}<0.01,{ }^{* * *} \mathrm{P}<0.001,{ }^{* * * *} \mathrm{P}<0.0001$.

A

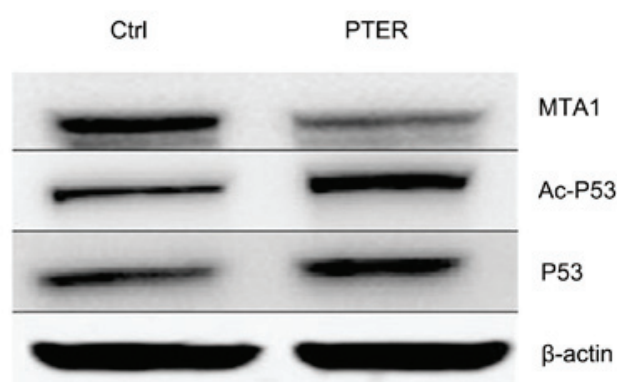

B

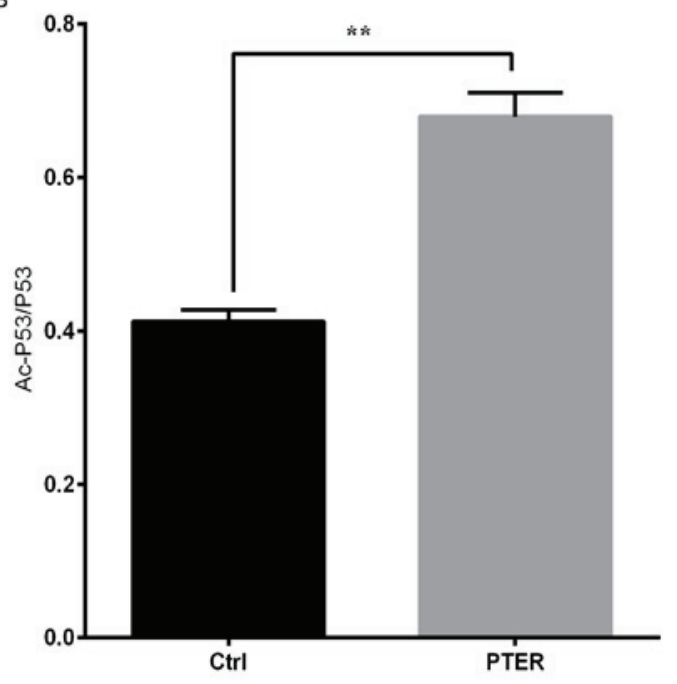

Figure 3. (A) Pterostilbene (PTER) induced metastasis-associated protein 1 (MTA1) downregulation is concomitant with increased p53 acetylation in western blot. (B) The ratio of Acp53 to total p53 was calculated by Image $\mathrm{J}$ software and the means \pm standard error of three independent experiments were shown. ${ }^{* *} \mathrm{P}<0.01$. Ctrl, control.

compared to the control group $(3.62 \pm 0.18$ and $4.41 \pm 0.30 \%)$ after treatment for 3 and 5 days, respectively, in SMMC7721 cells. Thus, PTER treatment can increase the apoptosis of SMMC7721 cells.

\section{Discussion}

MTA1, HDAC1 and HDAC2 belong to the NuRD co-repressor complex, which is related to the deacetylation of histones and other proteins and contributes to the specificity of transcriptional regulation. MTA1 is crucial to the expression of oncogenic proteins, as excess MTA1 expression is closely related to aggressive tumors $(8,9)$. It has been demonstrated that MTA1 can promote survival, prevent apoptosis, and increase invasion properties in prostate cancer (10).

In a previous study, we found that PTER induced the degradation of MTA1, which then destabilized the deacetylation of the MTA1/HDAC/NuRD complex, resulting in increased activation and acetylation of $\mathrm{p} 53$ in prostate cancer cells (10-13). In our present study, we proposed that PTER may 


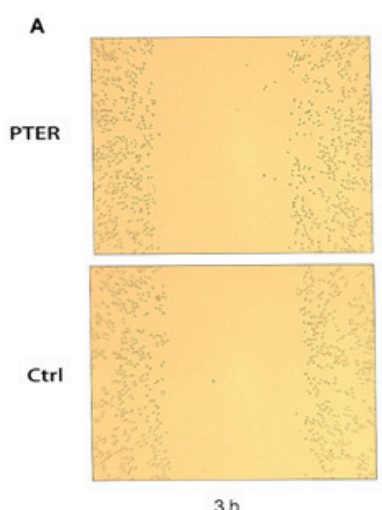

$3 \mathrm{~h}$

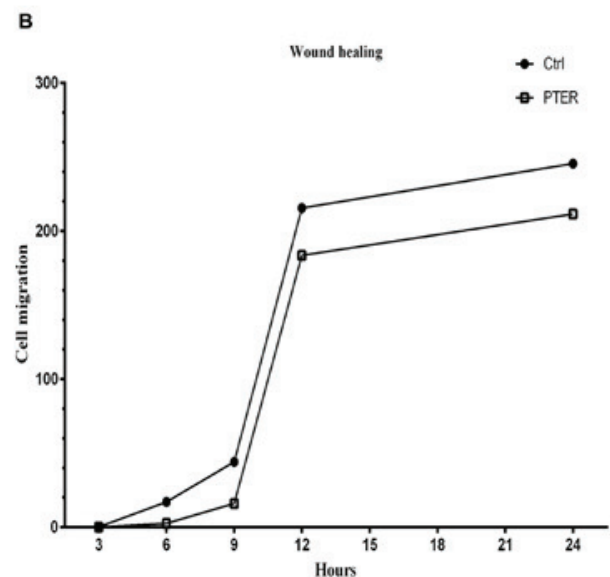

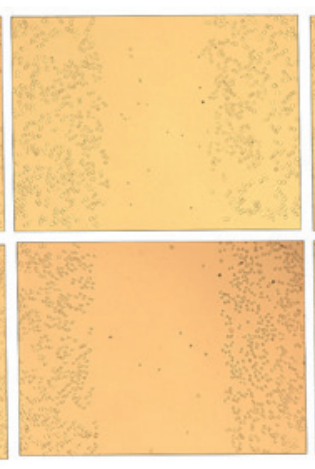

$9 \mathrm{~h}$

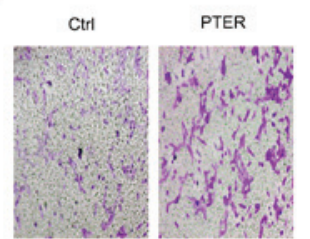

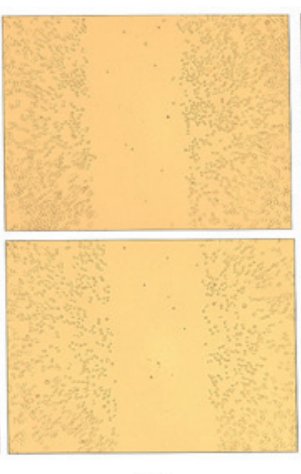

$12 \mathrm{~h}$

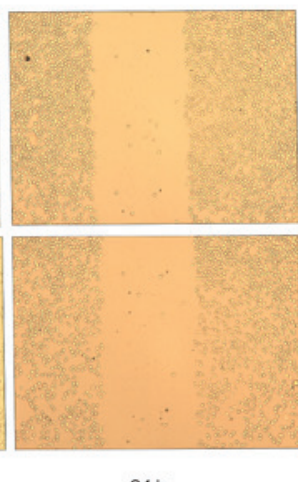

D

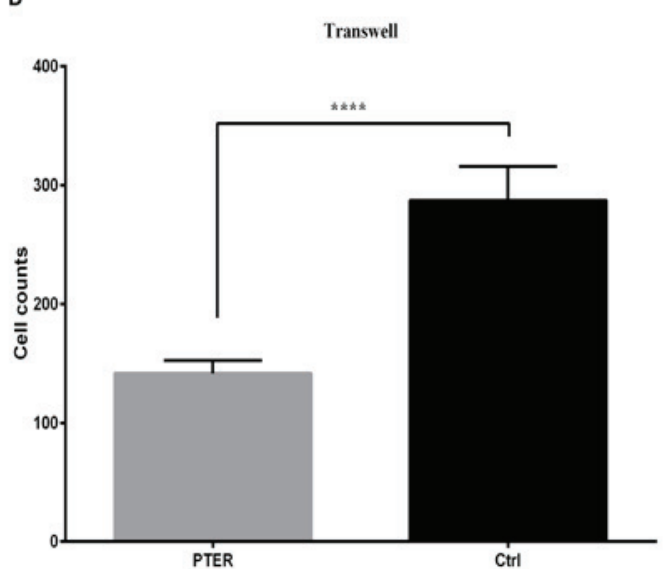

Figure 4. Pterostilbene (PTER) inhibited cell motility and invasion in SMMC7721 cells. (A) PTER inhibited the motility of SMMC7721 cells. Cells were treated with PTER $(50 \mu \mathrm{M})$ or DMSO [control $(\mathrm{Ctrl}) 50 \mu \mathrm{M}$ ]. The wound healing assay was carried out to examine cell motility at the indicated time points $(3$, $6,9,12$, and $24 \mathrm{~h}$ ). (B) Cell motility potential is graphed; data were normalized to that of the control group. (C) PTER inhibited cell invasion. Cells were treated or not with PTER $(50 \mu \mathrm{M})$ for $24 \mathrm{~h}$. (D) Results were compared with those of the control group. Representative photographs are shown at $\mathrm{x} 40$ magnification. Error bars represent the means \pm standard error, $\mathrm{n}=3 ;{ }^{* * * * *} \mathrm{P}<0.0001$.

be able to suppress the expression of MTA1 and thus show higher antitumor efficacy in HCC.

Few data are available about the expression of MTA1 after treatment with PTER and the potential effects on cancer metastasis and invasion in HCC. According to our present findings, we found that the expression of MTA1 may be related to cell invasion, motility, apoptosis, and cell cycle progression in HCC.

PTER, a dimethylether, is a natural analogue of resveratrol that was discovered in blueberries, has a safety pharmacology profile as an oral drug and has been reported to be the most effective inhibitor of MTA1 $(14,15)$. According to our results, we showed that PTER both affected HCC cell viability by inhibiting MTA1 and modulated MTA1-induced specificity of transcriptional gene regulation, including effects on Ac-p53. We revealed a novel epigenetic mechanism: The reduction in MTA1 led to inhibition of MTA1-HDAC complexes, which inhibited the deacetylase function, leading to increasing amounts of acetylation and modulation of the acetylation of p53 and promotion of the activation of apoptosis genes $(13,16)$. Therefore, MTA1 represents a novel PTER target that plays an important role in the clinical application of chemotherapy $(17,18)$. In our present study, we observed the downregulation of MTA1, HDAC1 and HDAC2 expression in PTER-treated cells. The ratio of Ac-p53-to-p53 was obviously higher after PTER treatment in tumors. Together, our findings indicated that PTER decreased the expression of MTA1, then destabilized MTA1/HDAC complexes to allow acetylation and activation of p53. The activation of p53 induced many anti-tumor mechanisms, which have critical effects on apoptosis, chromosome stability and the prevention of angiogenesis. When DNA is damaged, it can activate DNA repair proteins, and when DNA is irreversibly damaged, it can trigger cell programmed apoptosis (19). In parallel, PTER downregulated HDAC, and the relative imbalance of histone acetyltransferase (HAT) to HDAC enzyme activities increased the amount of HAT-induced acetylation (20). Increased p53 acetylation by PTER contributed to the transcriptional activation of genes linked with apoptosis and cycle arrest, resulting in inhibited HCC cell growth.

PTER is an effective anti-proliferative drug at concentrations up to $50 \mu \mathrm{M}$ and can sharply inhibit cell proliferation. According to our present study, PTER can induce cell cycle arrest in $\mathrm{S}$ phase and reduce the numbers of cells in $\mathrm{G} 2 / \mathrm{M}$ phase. At concentrations greater than $50 \mu \mathrm{M}$, PTER significantly induced apoptosis in SMMC7721 cells. Additionally, PTER affected cell motility and invasion at low concentrations of $\sim 50 \mu \mathrm{M}$.

This present study revealed that PTER had a significant inhibitory effect on cell proliferation, invasion, motility and 
A
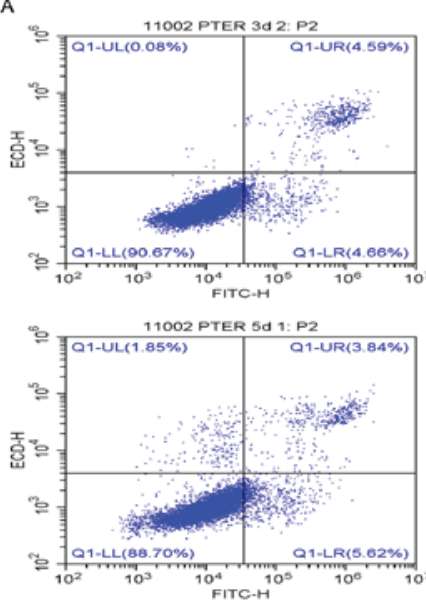

C
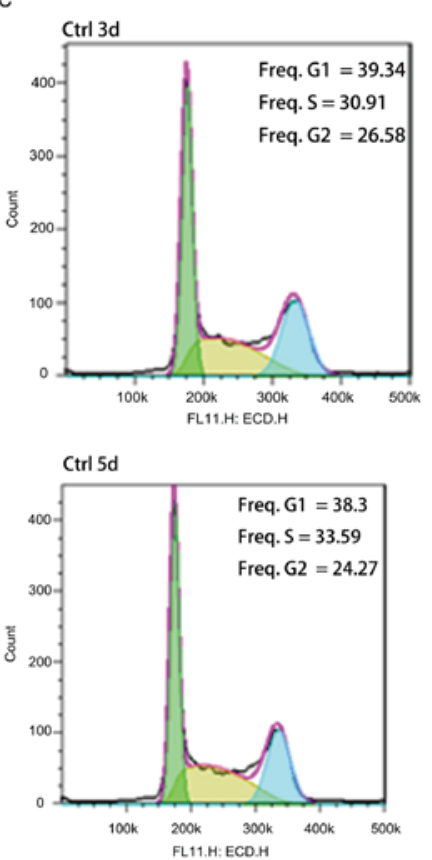
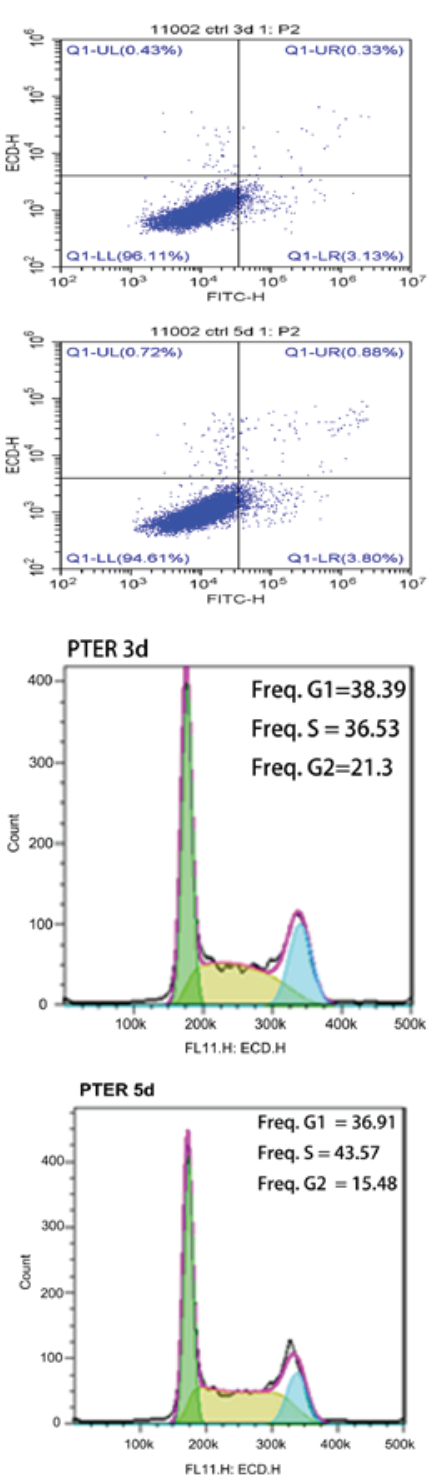

B
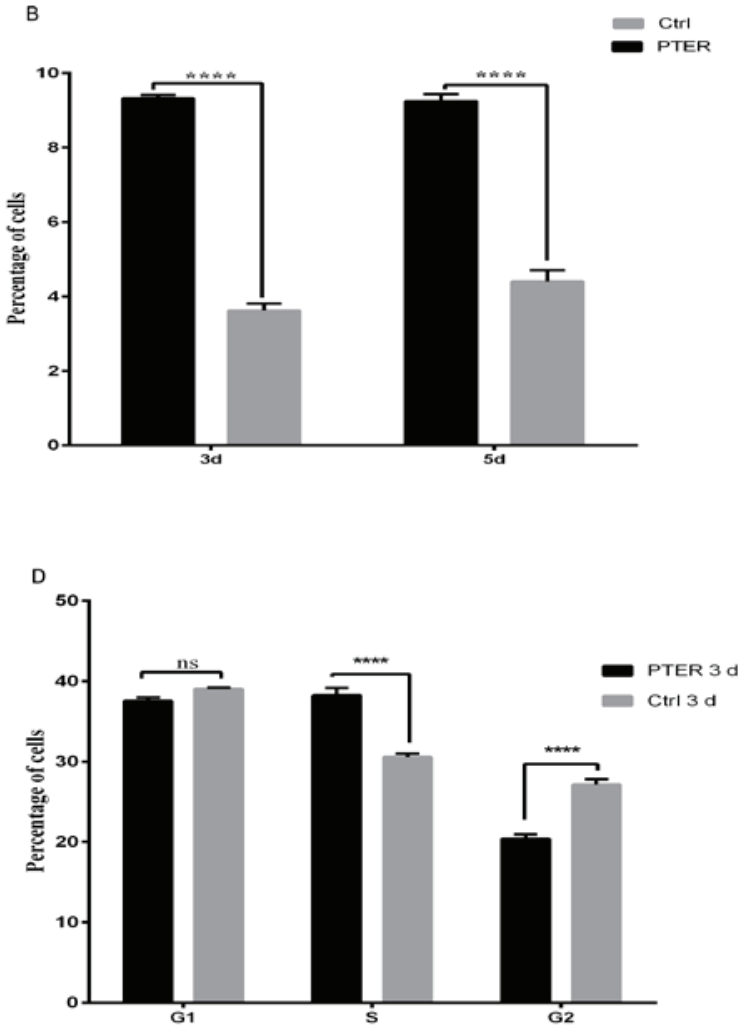

$E$

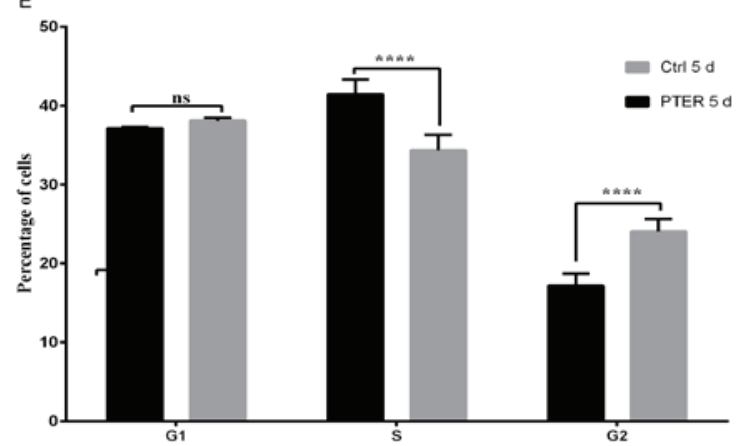

Figure 5. Pterostilbene (PTER) induced apoptosis and cell cycle arrest in SMMC7721 cells. (A) Cells were treated with or without PTER (50 $\mu$ M) for 3 or 5 days to detect apoptosis. (B) Apoptosis was analyzed by flow cytometry. (C) PTER induced cell cycle arrest in SMMC7721 cells at the G2/M phase. Flow cytometry analysis of SMMC7721 cells treated with DMSO [control (Ctrl)] or PTER (50 $\mu$ M) for 3 or 5 days. (D and E) SMMC7721 cells were analyzed by flow cytometry; representative images were obtained from flow cytometry assays, and the fractions of cells in G1, S and G2/M phases were semi-quantified. Values represent means \pm standard deviation of three independent experiments; ${ }^{* * * *} \mathrm{P}<0.0001$. ns, no significance.

apoptosis. Notably, it induced S phase arrest in SMMC7721 cells in vitro, which makes it a prospective therapeutic target drug for the treatment of liver cancer. Therefore, we also investigated the regulatory anticancer mechanism of PTER.

Our study demonstrated that PTER reduces the expression of MTA1, then destabilizes the MTA1/HDAC complex to allow the acetylation and activation of p53. This molecular mechanism may be involved in PTER-induced changes in cell proliferation, invasion, motility, apoptosis and cell cycle progression. PTER reduced the protein levels and enzymatic activities of MTA1 and HDAC, which inhibited motility and invasion in SMMC7721 cells.

In conclusion, this present in vitro study of PTER revealed its prospective anticancer properties, which may inspire future experiments, including (1) toxicity profiling and pharmacokinetics, (2) the generation of MTA1 gene knock-out models and (3) studies of the curative effect of anticancer treatment in HCC mouse models. MTA1 expression may be related to proliferation, invasion, motility, apoptosis, and cell cycle progression in HCC. We demonstrated that PTER could inhibit HCC growth and was an effective regulator of MTA1/HDAC-mediated p53 acetylation, apoptosis, motility and invasion in HCC cells. PTER inhibited cell proliferation, induced cell cycle arrest, triggered apoptosis and inhibited motility and invasion in human liver cancer cells. Our study of this novel epigenetic signaling pathway provided strong evidence for MTA1 as an effective clinical therapeutic target and novel anticancer agent in HCC. Finally, our research revealed a novel epigenetic signaling pathway of PTER that may provide new opportunities for the development of 
primary chemoprevention and chemotherapy approaches in HCC.

This study does not involve experiments performed on human participants or animals by any of the authors.

Informed consent was obtained from all individual participants included in this study.

\section{References}

1. Jemal A, Bray F, Center MM, Ferlay J, Ward E and Forman D: Global cancer statistics. CA Cancer J Clin 61: 69-90, 2011.

2. Ide T, Miyoshi A, Kitahara K and Noshiro H: Prediction of postoperative complications in elderly patients with hepatocellular carcinoma. J Surg Res 185: 614-619, 2013.

3. Paul S, DeCastro AJ, Lee HJ, Smolarek AK, So JY, Simi B, Wang CX, Zhou R, Rimando AM and Suh N: Dietary intake of pterostilbene, a constituent of blueberries, inhibits the beta-catenin/p65 downstream signaling pathway and colon carcinogenesis in rats. Carcinogenesis 31: 1272-1278, 2010.

4. Dias SJ, Zhou X, Ivanovic M, Gailey MP, Dhar S, Zhang L, He Z, Penman AD, Vijayakumar S and Levenson AS: Nuclear MTA1 overexpression is associated with aggressive prostate cancer, recurrence and metastasis in African Americans. Sci Rep 3: 2331,2013

5. Wilson MA, Rimando AM and Wolkow CA: Methoxylation enhances stilbene bioactivity in Caenorhabditis elegans. BMC Pharmacol 8: 15, 2008.

6. Kai L, Wang J, Ivanovic M, Chung YT, Laskin WB, Schulze-Hoepfner F, Mirochnik Y, Satcher RL Jr and Levenson AS: Targeting prostate cancer angiogenesis through metastasis-associated protein 1 (MTA1). Prostate 71: 268-280, 2011.

7. Kai L, Samuel SK and Levenson AS: Resveratrol enhances p53 acetylation and apoptosis in prostate cancer by inhibiting MTA1/NuRD complex. Int J Cancer 126: 1538-1548, 2010.

8. Denner DR and Rauchman M: Mi-2/NuRD is required in renal progenitor cells during embryonic kidney development. Dev Biol 375: 105-116, 2013.

9. Dannenmann C, Shabani N, Friese K, Jeschke U, Mylonas I and Brüning A: The metastasis-associated gene MTA1 is upregulated in advanced ovarian cancer, represses ER $\beta$, and enhances expression of oncogenic cytokine GRO. Cancer Biol Ther 7: 1460-1467, 2008.
10. Li K, Dias SJ, Rimando AM, Dhar S, Mizuno CS, Penman AD, Lewin JR and Levenson AS: Pterostilbene acts through metastasis-associated protein 1 to inhibit tumor growth, progression and metastasis in prostate cancer. PLoS One 8: e57542, 2013.

11. Dhar S, Kumar A, Li K, Tzivion G and Levenson AS: Resveratrol regulates PTEN/Akt pathway through inhibition of MTA1/HDAC unit of the NuRD complex in prostate cancer. Biochim Biophys Acta 1853: 265-275, 2015.

12. Levenson AS, Kumar A and Zhang X: MTA family of proteins in prostate cancer: Biology, significance, and therapeutic opportunities. Cancer Metastasis Rev 33: 929-942, 2014

13. Dias SJ, Li K, Rimando AM, Dhar S, Mizuno CS, Penman AD and Levenson AS: Trimethoxy-resveratrol and piceatannol administered orally suppress and inhibit tumor formation and growth in prostate cancer xenografts. Prostate 73: 1135-1146, 2013.

14. Kapetanovic IM, Muzzio M, Huang Z, Thompson TN and McCormick DL: Pharmacokinetics, oral bioavailability, and metabolic profile of resveratrol and its dimethylether analog, pterostilbene, in rats. Cancer Chemother Pharmacol 68: 593-601, 2011.

15. Colaluca IN, Tosoni D, Nuciforo P, Senic-Matuglia F, Galimberti V, Viale G, Pece S and Di Fiore PP: NUMB controls p53 tumour suppressor activity. Nature 451: 76-80, 2008.

16. Manavathi B, Singh K and Kumar R: MTA family of coregulators in nuclear receptor biology and pathology. Nucl Recept Signal 5: e010, 2007.

17. Tang Y, Zhao W, Chen Y, Zhao Y and Gu W: Acetylation is indispensable for p53 activation. Cell 133: 612-626, 2008.

18. McCubrey JA and Demidenko ZN: Recent discoveries in the cycling, growing and aging of the p53 field. Aging Albany NY 4: 887-893, 2012.

19. Shirai O, Ohmiya N, Taguchi A, Nakamura M, Kawashima H, Miyahara R, Itoh A, Hirooka Y, Watanabe O, Ando T, et al: $\mathrm{P} 53, \mathrm{p} 21$, and $\mathrm{p} 73$ gene polymorphisms in gastric carcinoma. Hepatogastroenterology 57: 1595-1601, 2010.

20. Prasad R and Katiyar SK: Polyphenols from green tea inhibit the growth of melanoma cells through inhibition of class I histone deacetylases and induction of DNA damage. Genes Cancer 6: 49-61, 2015. 